\title{
Cost and length of hospital stay for healthcare facility- onset Clostridioides Difficile infection in pediatric wards: a prospective cohort analysis
}

\author{
Sevliya Öcal Demiri ${ }^{1 \oplus}$, Eda Kepeneklii ${ }^{2 \oplus}$, Gülşen Akkoç ${ }^{3 \oplus}$, Nurhayat Yakut ${ }^{4 \oplus}$, \\ Ahmet Soysal ${ }^{5 \odot}$
}

${ }^{1}$ Division of Pediatric Infectious Diseases, Department of Pediatrics, İstanbul Medeniyet University Göztepe Prof. Dr. Süleyman Yalçın City Hospital, İstanbul; ${ }^{2}$ Division of Pediatric Infectious Diseases, Department of Pediatrics, Marmara University Faculty of Medicine, İstanbul; ${ }^{3}$ Division of Pediatric Infectious Diseases, University of Health Sciences, İstanbul Haseki Training and Research Hospital, İstanbul; ${ }^{4}$ Clinic of Pediatric Infectious Diseases, Başakşehir Çam and Sakura City Hospital, İstanbul; ${ }^{5}$ Clinic of Pediatric Infectious Diseases, Memorial Ataşehir Hospital, İstanbul, Turkey.

\begin{abstract}
Background. Clostridioides difficile (C. difficile) is a well-known causative agent of healthcare associated infection, it increases medical cost besides increasing morbidity and mortality. This study was conducted to determine the incidence, and economic burden of healthcare facility-onset C. difficile infection (HO-CDI) in children.
\end{abstract}

Methods. Data was acquired with a prospective cohort study conducted in pediatric wards of a tertiary university hospital between August 2015 to August 2016. The HO-CDI was defined as diarrhea that began after 48 hours of admission with a positive cytotoxic stool assay for the presence of toxin A and/or B of C. difficile.

Results. In the 3172 admissions in one year, 212 (7\%) healthcare associated diarrhea (HAD) episodes were observed, in 25 (12\%) of them C. difficile was identified in which 6 (25\%) cases <2-year-old. The incidence of HOCDI was estimated as 8.8/10,000 patient-days. Cases with HO-CDI ( $n=19)$ were compared with cases with nonCDI-HAD ( $\mathrm{n}=102)$; the presence of one of the risk factors for CDI increased the risk for HO-CDI $(5,05 ; 95 \% \mathrm{Cl}$ : 1.10-23.05; P 0,037), the median length of stay (LOS) attributable HO-CDI was 7 days (IQR,5-10) per admission, whereas for non-CDI-HAD was 2 days $(\mathrm{IQR}, 0-4)(\mathrm{p}=0.036)$. General hospitalization costs in the two groups were similar, specifically estimated costs attributable to HO-CDI and non-CID-HAD were $\$ 294.0$ and $\$ 137.0$ per hospitalization respectively $(\mathrm{p}=<0.0001)$.

Conclusion. Although in children the incidence of HO-CDI is increasing, its clinical manifestation is still milder and effective infection control measures with antibiotic stewardship can limit related morbidly, mortality, LOS, and cost.

Key words: health-care, HO-CDI, C. difficile, cost, pediatric.

Clostridioides (formerly Clostridium) difficile (C. difficile) is an anaerobic, gram-positive, toxinproducing bacillus, which exists in spore form in the environment and is a member of the human gastrointestinal system. During or following the usage of broad-spectrum antibiotics, colonic microbiota is disrupted and C. difficile

$凶$ Sevliya Öcal Demir

sevliyademir@gmail.com

Received 10th November 2020, revised 27th February 2021, accepted 27th April 2021. starts to multiply and produce toxins leading to diarrhea. Particularly hospitalized elderly patients with comorbidities are more vulnerable to $C$. difficile infection (CDI) than children. ${ }^{1}$ But the incidence and severity of CDI is gradually increasing in children. ${ }^{2}$ In the United States its incidence in children $<18$ years was reported as 24.2 cases per 100,000 population in 2011 . $^{3}$ In Turkey data about pediatric CDI are very limited, Karaaslan et al. ${ }^{4}$ reported the incidence of CDI in hospitalized children as 9 per 1000 patients for the years 2013 and 2014. 
Healthcare associated CDI is associated with prolonged length of hospital stay (LOS), readmission, extra healthcare cost, increased morbidity and mortality. Recent studies found that the attributable cost of CDI ranges from $\$ 3,000$ to $\$ 15,000$ per hospitalization and LOS of 3-7 days in hospitalized adults. ${ }^{5-7}$ Only one study has evaluated the impact of CDI in hospitalized children, and they reported the attributable cost of HO-CDI to range from $\$ 1,917$ to $\$ 8,317$, and attributable LOS as approximately 4 days. ${ }^{7}$

For there is no prospective study that specifically evaluated additional LOS and attributable cost of HO-CDI in children, we conducted this study to determine the incidence, LOS and healthcare cost attributable of HO-CDI in pediatric patients.

\section{Material and Methods}

This prospective cohort study was carried out from August 12015 till July 312016 at the pediatric wards of Marmara University Medical School. This hospital was founded in January 2011 with a 649 bed-capacity, our patients are of a middle socioeconomic statue. This investigation was conducted at our pediatric department which included patients from the pediatric intensive care unit (PICU) with a 14 bed capacity, the pediatric hematologyoncology unit with a 27 bed capacity, and the general pediatric ward with a 77 bed- apacity.

During 12 months, all admissions of pediatric patients were followed and those aged between 2 to 18 years who were hospitalized for more than 48 hours were recorded daily by one Pediatric Infectious Diseases specialist. Data concerning the patient's age, sex, diagnosis, previous hospitalization, type number and duration of antibiotics used in last 3 months, other medications including chemotherapy, proton pump inhibitors (PPIs), diagnostic tests and treatment for CDI and their costs, duration of hospitalization, outcomes and total hospitalization cost was documented in a data collection sheet.
Health associated diarrhea (HAD) was defined according to the Center for Diseases Control and Prevention (CDC) criteria; diarrhea $\geq 3$ loose or looser-than-normal stools in a 24-hour, of $<7$ days duration period began after 48 hours of hospitalization. CDI was defined according to the guidance and recommendations from the Infectious Diseases Society of America (IDSA) and the Society for Healthcare Epidemiology of America (SHEA); the presence of symptoms (mainly diarrhea) and either a stool test positive for $C$. difficile toxins or detection of toxigenic C. difficile, or colonoscopic or histopathologic findings revealing pseudomembranous colitis. Detection of toxins $\mathrm{A}$ and $\mathrm{B}$ of $\mathrm{C}$ difficile was performed by using premier toxins $\mathrm{A}$ and $\mathrm{B}$ (C. difficile) EIA kit bioMeriux (Marseile, France) according to the manufacturer's instructions. To increase comparability between clinical settings, we used IDSA recommendation for case definition, incidence estimation were used for standardized case definition. ${ }^{8}$ To evaluate the impact of CDI on hospitalization cost and LOS, patients with HAD were designated into two groups, the first group had healthcarefacility onset (HO)-CDI (HO-CDI), the other had HAD without CDI (non-CDI-HAD). Because asymptomatic colonization with $C$ difficile is common in the neonatal period and infancy, ${ }^{9-11}$ children $<2$-year-old were excluded from the two groups. Firstly, general hospitalization cost was estimated by including all costs associated with hospitalization, and compared the two groups. During the patient stay in pediatric wards, their cost and outcomes were tracked. The specific cost attributable to CDI was calculated with the charge for inpatients, closed beds for isolation, laboratory tests, antimicrobial drugs and other medications used for CDI. Inpatient cost of ICU was not added as an extra cost, because HAD was mild in all and PICU stay was not due to HAD. Also, for other patients when their stay was for their primer disease instead of HAD bed cost was not added as an extra cost. The cost was first recorded in Turkish Lira (TL), then converted to USD (\$), using the average exchange rate between TL to USD currency between 1 August 2015 to 31 July 2016 (1TL 
$=0.3424 \$)$. Attributable LOS associated with HAD was estimated after a daily patient visit to clarify if the patient stayed due to HAD.

The study protocol was approved by the decision of the Clinical Research Ethics Committee of Marmara University Medical School (number: 09.2015.221).

\section{Statistical analysis}

Data were analyzed using SPSS version 21 (SPSS Inc., Chicago, IL, USA). Frequency and percentage for categorical data, and median (inter quarter range) for continuous data were identified as descriptive statistics. Mann Whitney U-test was used to compare the two groups. Logistic regression analysis was used to analyze risk factors. The results were considered statistically significant in cases that p-value is less than 0.05 .

\section{Results}

Between August 12015 and July 312016 1,971 patients had 3,172 admissions for more than 48 hours in pediatric units under surveillance. The repeated admissions mainly belonged to the hematology-oncology patients. During a 12 month follow-up 212 HAD episodes were observed; in 150 (70,75\%) the microbiologic agents could not be identified, in 25 C. difficile, in 23 rotaviruses, in 7 Giardia intestinalis, in 3 adenoviruses, and in 3 Entamoeba intestinalis was identified. Because none of our patients had severe diarrhea and some of them were neutropenic colonoscopic examination was not done. CDI was defined according to a positive stool test for $C$. difficile toxins in all of the patients. To estimate HO-CDI incidence, the cases <2-year-old were excluded, the number of cases with HO-CID decreased to 19, total patient-days decreased to 21,520 . The incidence of HO-CDI was found as $8,8 / 10,000$ patientdays in children aged between 2-18 years. Other characteristics of our sample group are summarized in Table I.
The distribution of demographic-clinic characteristics and risk factors among the 19 cases with HO-CDI and 102 cases with nonCDI-HAD are shown in Table II. Statistical analysis showed the presence of any of the following risk factors including enteral feeding, PPI, gastrostomy, chemotherapy, immune suppression other than antibiotic usage for CDI increased the risk for HO-CDI 5-fold (5,05; 95\% Cl: 1.10-23.05). Antibiotic exposure in the previous 3 months in HO-CDI and non-CDI-HAD groups were $\% 84,2$ and $\% 88$ respectively, similarly total antibiotic days, type of antibiotics used, were not different in the two groups (Table II), (Fig. 1). Also, repeated hospitalization, hospitalization in the PICU, being a hematology/oncology patient with a malignancy were not statistically different in the two groups.

Table I. Demographic characteristics of hospitalized pediatric cases.

\begin{tabular}{|c|c|}
\hline & $\begin{array}{c}\text { Median (IQR) or } \\
\text { N }(\%)\end{array}$ \\
\hline Total number of patients* $(\mathrm{n})$ & 1,971 \\
\hline $\begin{array}{l}\text { Total number of hospital } \\
\text { admission* }(n)\end{array}$ & 3172 \\
\hline Boys & $1,105(56.1)$ \\
\hline Girls & $866(43.9)$ \\
\hline Age, months & $48,0(12.0-108.0)$ \\
\hline Total hospitalization days, $\mathrm{n}=1971$ & 31114 \\
\hline Mean hospitalization days & $6,0(4.0-11.0)$ \\
\hline Hospitalized in $(n=1971)$ & 1971 \\
\hline PICU** & $113(5.7)$ \\
\hline $\begin{array}{l}\text { Hematology-oncology ward + } \\
\text { PICU }\end{array}$ & $14(0.7)$ \\
\hline Hematology-oncology ward & $346(17.6)$ \\
\hline General wards + PICU & $56(2.8)$ \\
\hline General pediatric ward & $1442(73.2)$ \\
\hline $\begin{array}{l}\text { Healthcare associated diarrhea } \\
\text { (HAD) episodes }\end{array}$ & 212 \\
\hline C. difficile & 25 \\
\hline Rotaviruses & 23 \\
\hline Giardia intestinalis & 7 \\
\hline Adenoviruses & 3 \\
\hline Entamoeba intestinalis & 3 \\
\hline Non-identified & 151 \\
\hline
\end{tabular}


Table II. Distribution of demographic and clinic characteristics, risk factors in the pediatric cases with HO-CDI or non-CDI-HAD.

\begin{tabular}{|c|c|c|c|c|}
\hline \multicolumn{2}{|c|}{ Characteristics, N=121 } & $\begin{array}{c}\text { CDI } \\
\mathrm{n}=19 \\
\mathrm{~N}(\%) \text { or Median } \\
(\mathrm{IQR})\end{array}$ & $\begin{array}{c}\text { Non-CDI } \\
\mathrm{n}=102 \\
\mathrm{~N} \text { (\%) or Median } \\
\text { (IQR) }\end{array}$ & $\mathrm{p}$ \\
\hline \multirow{2}{*}{ Gender } & Male & $9(47.4)$ & $68(66.7)$ & 0.125 \\
\hline & Female & $10(52.6)$ & $34(33.3)$ & \\
\hline \multirow[t]{3}{*}{ Age, months } & & $48(36-108)$ & $48(35-96)$ & 1.000 \\
\hline & PICU & $2(10.5)$ & $4(3.9)$ & 0.487 \\
\hline & Hematology-oncology ward + PICU & $1(5.3)$ & $10(9.8)$ & \\
\hline \multirow[t]{3}{*}{ Hospitalized in } & General wards + PICU & $2(10.5)$ & $8(7.8)$ & \\
\hline & Hematology-oncology ward & $9(47.4)$ & $37(36.3)$ & \\
\hline & General pediatric ward & $5(26.3)$ & $43(42.2)$ & \\
\hline \multicolumn{2}{|c|}{ Recurrent hospitalization } & $13(68.4)$ & $52(51.0)$ & 0.212 \\
\hline \multicolumn{2}{|c|}{ Length of stay (LOS), (day) } & $22(7-44)$ & $16(10-29)$ & 0.556 \\
\hline \multicolumn{2}{|c|}{ Duration of PICU hospitalization, (day) } & 29 (13-93), n:5 & $10(6-28), \mathrm{n}: 29$ & 0.871 \\
\hline \multicolumn{2}{|c|}{ Diarrhea onset day of hospitalization, (day) } & $9(2-20)$ & $6(3-14)$ & 0.634 \\
\hline \multicolumn{2}{|c|}{ Duration of diarrhea } & $4(3-6)$ & $3(3-5)$ & 0.109 \\
\hline \multicolumn{2}{|c|}{ Presence of any risk factor for HAD } & $17(89.5)$ & $64(62.7)$ & 0.032 \\
\hline \multicolumn{2}{|l|}{ Chemotherapy } & $11(57.9)$ & $39(38.2)$ & 0.132 \\
\hline \multicolumn{2}{|l|}{ Nasogastric tube } & $2(10.5)$ & $13(12.7)$ & 1.000 \\
\hline \multicolumn{2}{|l|}{ PPI } & $6(31.6)$ & $17(16.7)$ & 0.198 \\
\hline \multicolumn{2}{|l|}{ Gastrostomy } & $1(5.3)$ & $7(6.9)$ & 1.000 \\
\hline \multicolumn{2}{|l|}{ IV Catheter } & $4(21.1)$ & $12(11.8)$ & 0.277 \\
\hline \multicolumn{2}{|c|}{ Immunosuppressive treatment } & - & $1(1.0)$ & 1.000 \\
\hline \multicolumn{2}{|c|}{ Immunodeficiency } & $1(5.3)$ & $3(2.9)$ & 0.500 \\
\hline \multirow{4}{*}{ Surgical operation } & None & $17(89.5)$ & $98(96.1)$ & 0.261 \\
\hline & Cranial & $2(10.5)$ & $2(2.0)$ & \\
\hline & Abdomen & - & $1(1.0)$ & \\
\hline & Head/Neck & - & $1(1.0)$ & \\
\hline \multicolumn{2}{|c|}{ Antibiotic, anti-viral and anti-fungal usage } & $16(84.2)$ & $88(86.3)$ & 0.730 \\
\hline \multicolumn{2}{|c|}{ Total antibiotic days } & $15(8-31)$ & $13(5-32)$ & 0.797 \\
\hline \multicolumn{2}{|l|}{ Penicillins, (day) } & $14(9-17)$ & $11(5-16)$ & 0.495 \\
\hline \multicolumn{2}{|c|}{ Aminopenicillins, (day) } & $7(7-7)$ & $4(2-7)$ & 0.376 \\
\hline \multicolumn{2}{|c|}{ Piperacillin-tazobactam, (day) } & $8(5-13)$ & $6(4-10)$ & 0.262 \\
\hline \multicolumn{2}{|c|}{ Carbapenems, (day) } & 15 (10-19) & $10(5-14)$ & 0.535 \\
\hline \multicolumn{2}{|l|}{ Macrolides, (day) } & $9(9-9)$ & $10(2-10)$ & 0.450 \\
\hline Cephalosporins, ( & lay) & $8(5-11)$ & $5(2-10)$ & 0.111 \\
\hline Glycopeptides-lin & zolid, (day) & $10(8-14)$ & $10(4-14)$ & 0.393 \\
\hline Aminoglycosides, & (day) & $8(2-8)$ & $3(1-10)$ & 0.215 \\
\hline Quinolones, (day) & & $8(8-8)$ & $7(3-12)$ & 0.788 \\
\hline Colistin, (day) & & $9(6-10)$ & $5(4-6)$ & 0.119 \\
\hline TM-SMX, (day) & & - & $12(8-19)$ & 0.382 \\
\hline Anti-virals, (day) & & $7(5-8)$ & $11(6-20)$ & 0.647 \\
\hline Anti-fungals, (day & & $10(10-10)$ & $11(6-20)$ & 0.197 \\
\hline Mortality & No & $18(94.7)$ & $95(93,1)$ & 1.000 \\
\hline Mortality & Yes & $1(5.3)$ & $7(6.9)$ & \\
\hline Additional treatm & nt usage & $18(94.7)$ & $24(23.5)$ & $<0.0001$ \\
\hline
\end{tabular}




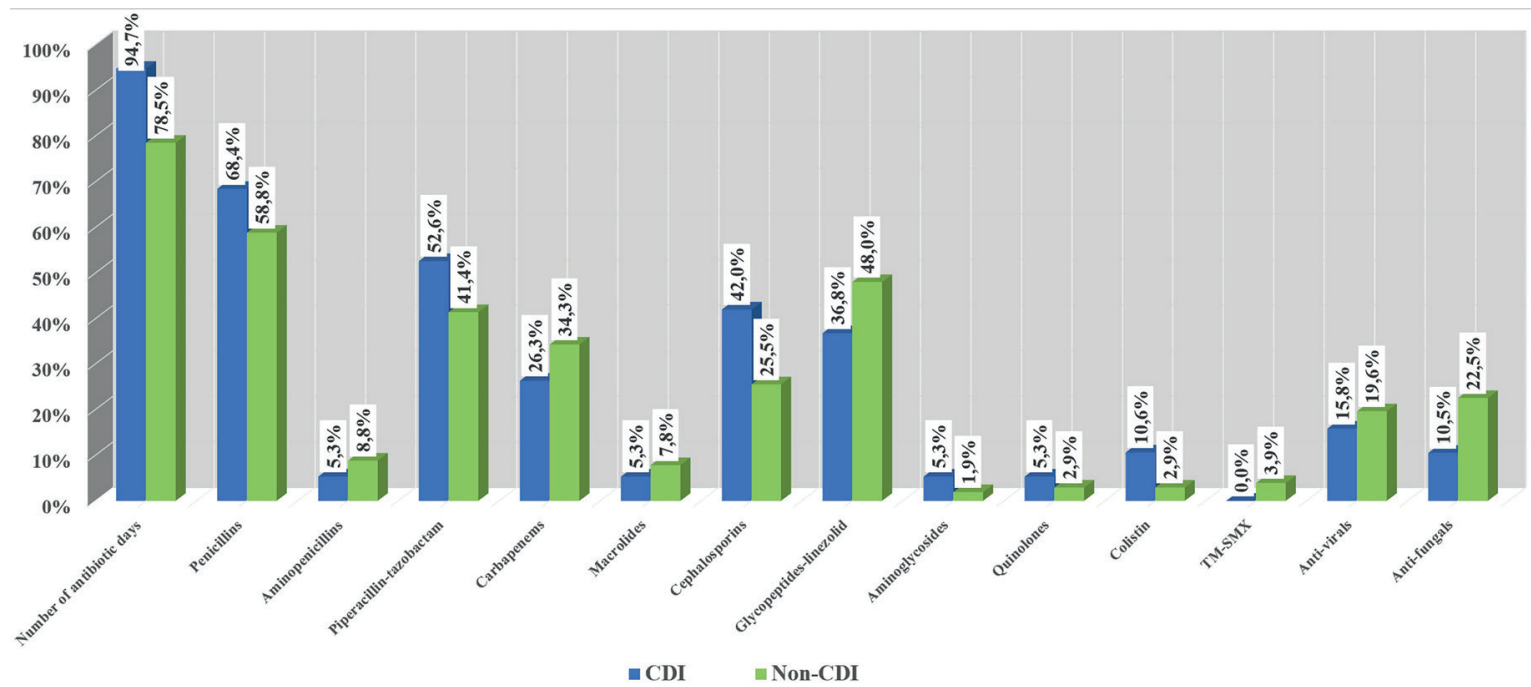

HO-CDI: healthcare facility-onset Clostridioides difficile infection, non-CDI-HAD: non- Clostridioides difficile infection health care associated diarrhea, CDI: Clostridioides difficile infection.

Fig. 1. Type of antibiotics usage in HO-CDI and non-CDI-HAD.

Table III. LOS and extra cost attributable to HO-CDI and non-CDI-HAD.

\begin{tabular}{lccc}
\hline Cost & $\begin{array}{c}\text { HO-CDI } \\
(\mathrm{n}=19)\end{array}$ & $\begin{array}{c}\text { Non-CDI-HAD } \\
(\mathrm{n}=102)\end{array}$ & $\mathrm{p}$ \\
\hline Total hospitalization cost $(\$)$ & $7.807(1.548-11.610)$ & $7.311(830-9.763)$ & 0.847 \\
Specifically, estimated costs for & $231.0(110.0-318.0)$ & $61.0(0.0-194.0)$ & $<0.0001$ \\
Hospitalization $(\$)$ & $70.0(24.0-76.0)$ & $51.0(23.0-49.0)$ & 0.149 \\
Diagnosis (\$) & $28.0(13.0-36.0)$ & $25.0(10.0-26.0)$ & 0.771 \\
Treatment Cost (\$) & $294.0(163.0-405.0)$ & $137.0(54.0-180.0)$ & $<0.0001$ \\
Total $(\$)$ & $7(5-10)$ & $2(0-4)$ & $<0.036$ \\
Length of stay (LOS) & & & \\
\hline
\end{tabular}

The general total cost of hospitalization in cases with HO-CDI was $\$ 7,807$ and in cases with non-CDI-HAD was $\$ 7,311$, which were similar. On the other hand, specifically calculated cost attributable to HAD in cases with HO-CDI (total cost was \$294) was higher than in patients with non-CDI-HAD (total cost was \$137), this difference was statistically significant $(p=<$ 0.0001). (Table III). The distribution of the cost attributable to HO-CDI was demonstrated in Figure 2, inpatient cost was higher in HO-CDI.

The median LOS attributable HO-CDI was 7 days (IQR, 5-10) per admission, whereas for non-CDI-HAD was 2 days (IQR,0-4) $(\mathrm{p}=0.036)$. The mortality rate was not found to be different between the two groups.

\section{Discussion}

The diagnosis of CDI in pediatric patients can be challenging due to the high rates of asymptomatic colonization with $C$. difficile. It can be detected in $25-50 \%$ of neonates and $40-$ $70 \%$ of infants. ${ }^{12}$ This is the most important factor for not determining the true incidence of CID in children. To partially overcome this problem, children under 2-year-old were excluded from the analysis. In addition, the presence of predisposing conditions, such as antibiotic exposure, gastric acid suppression, malignancy in most of the patients supported the diagnosis. 


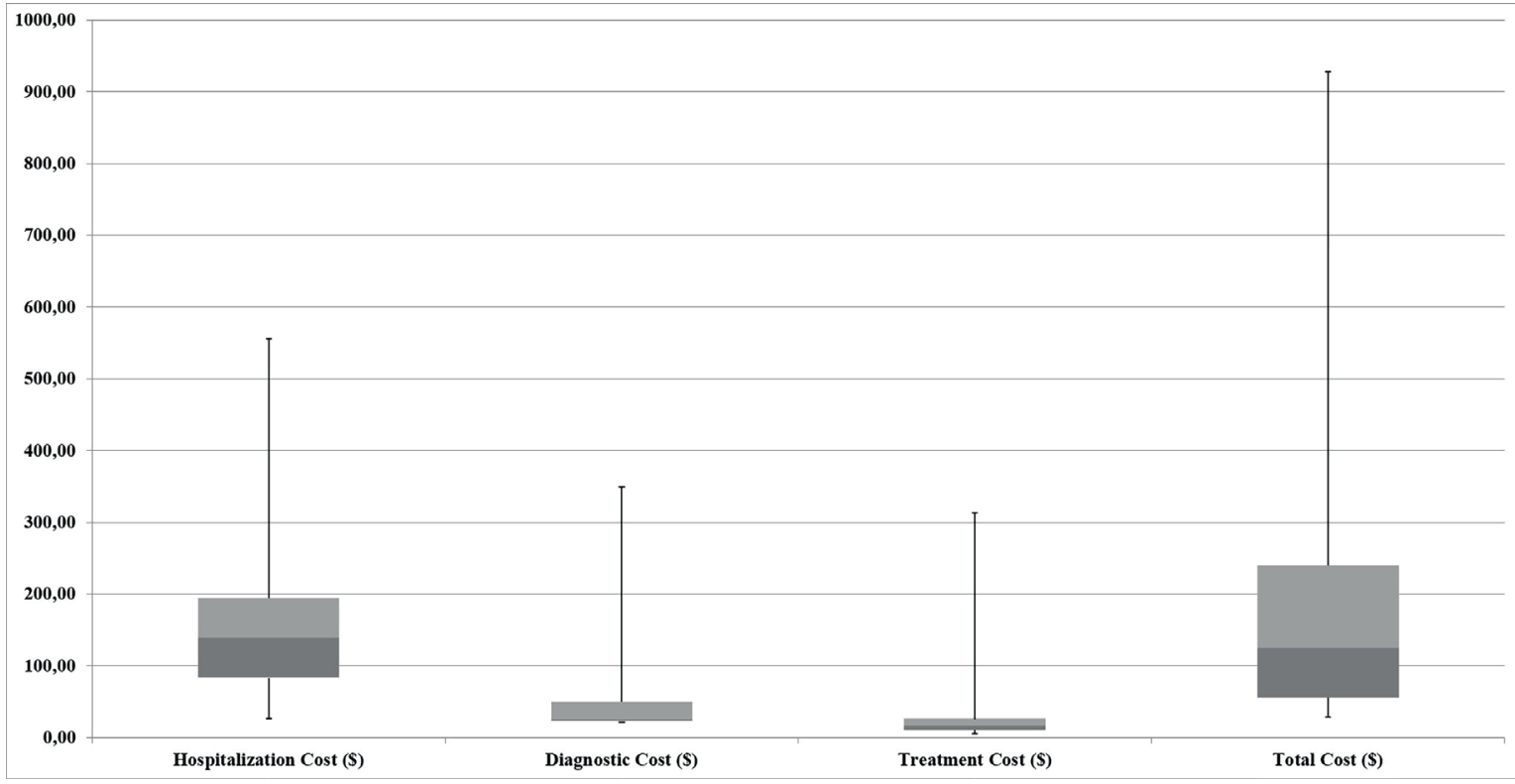

Fig. 2. Distribution of cost in pediatric patients with HO-CDI.

CDI is less frequent in children, the reported incidence was 24.2 cases per 100,000 pediatric population in the United States in 2011, but this was a population-based surveillance. ${ }^{3}$ Kukla et al. ${ }^{13}$ use case definition according to the IDSA recommendation and found HOCDI incidence as 2,4 cases/10,000 man-days. Here we found HO-CDI incidence as 8,8 cases per 10,000 patient-day. The incidence of CDI is increasing even in children. This result may be due to the fact that our hospital is a reference hospital, especially for hematology-oncology patients. Indeed, Kukla et al. ${ }^{13}$ stated that CID incidence is higher in reference hospitals than in community hospitals. A previous retrospective study from Turkey reported CDI incidence as 9 cases per 1000 patients in 2014 in hospitalized children. ${ }^{4}$

Previous antibiotic exposure is the well-known single most important risk factor for CDI, and in pediatric studies, multiple classes of antibiotics used in the preceding month has been associated with severe and recurrent CDI. ${ }^{14,15}$ In a recent study by Khalil et al. ${ }^{16}$ antibiotic usage and LOS were reported as predisposing factors for CDI. In this study, there was no difference concerning previous antibiotic exposure, type of antibiotics used and total antibiotic-days between the HO-CDI and non-CID-HAD groups. This may be because we compared HOCID with non-CID-HO-HAD, rather than cases without HAD, additionally clindamycin which is one of the most blamed antibiotics for CID was never used, fluoroquinolones were used infrequently. Factors such as enteral feeding, PPIs, gastrostomy, chemotherapy and immune suppression were shown to be risk factors for CDI in other studies; PPI usage and malignancy are the most common conditions among hospitalized children with CDI, accounting for 20 to $25 \%$ of HO-CDI. ${ }^{17,18}$ In the current study, the presence of one of the risk factors defined for CDI, increased HO-CDI risk by 5-fold. But specific risk factors could not define, this result may be due to the small number of patients in the HO-CDI group.

The cost attributable to CDI is expected to be lower in pediatric patients because in children severe disease or complications are not as common as in adults. Indeed, in this study no CDI- related complication was observed. Studies of hospitalized adults with CDI have found related costs ranging from $\$ 3,000$ to $\$ 15,000$ per hospitalization and LOS of 3-7 days..$^{5-7}$ There was only one study conducted by Mehrotra et al. ${ }^{7}$ who evaluated the impact 
of CDI on LOS and costs in children and reported that the attributable cost of $\mathrm{HO}-\mathrm{CDI}$ ranged from $\$ 1,917$ to $\$ 8,317$, and attributable LOS as approximately 4 days. In our study we found HO-CDI related costs as \$294 per hospitalization. Although this cost is higher than in the non-CDI-HAD group, it is a small difference in contrast to costs determined by previous studies. Milder manifestation of HOCID in our cases can only partly explained this difference. The prospective design of our study allowed us to follow cases closely and distinguish CID related costs. Retrospective studies have limited clinical and laboratory data, where only the total cost of hospitalization has been obtained, control groups are created from the same population with similar characteristics to estimate attributable cost of CDI. Another limitation is that we only estimated direct medical cost, we could not add the cost of cleaning materials, gloves and gowns, time spent by doctors and nurses, indirect costs such as school absenteeism or parental leave from work.

As expected, HO-CDI resulted in an extension of the duration of hospitalization for ongoing diarrhea, complications associated with the CDI diagnosis or treatment. Studies supported this hypothesis; in hospitalized adults with CDI this time was reported as 3-7 days, likewise in children this is approximately 4 days. ${ }^{5-7}$ In our study LOS was similarly 7 days (IQR,5-10) and longer than for non-CID-HAD.

In conclusion, although in children the incidence of HO-CDI is increasing, its clinic is still milder and effective infection control measures and antibiotic stewardship should limit related LOS and cost.

\section{Author contribution}

The authors confirm contribution to the paper as follows: study conception and design: AS, SÖD; data collection: SÖD, NY, GA; analysis and interpretation of results: SÖD, AS, EK; draft manuscript preparation: SÖD. All authors reviewed the results and approved the final version of the manuscript.

\section{Ethical approval}

Study protocol was approved by the decision of Clinical Research Ethic Committee of Marmara University Medical School (number: 09.2015.221).

\section{Source of funding}

This research did not receive any specific grant from funding agencies in the public, commercial, or not-for-profit sectors.

\section{Conflict of interest}

All authors have no potential conflicts of interest to disclose.

\section{REFERENCES}

1. Adams DJ, Eberly MD, Rajnik M, Nylund CM. Risk factors for community-associated Clostridium difficile infection in children. J Pediatr 2017; 186: 105109. https://doi.org/10.1016/j.jpeds.2017.03.032

2. Deshpande A, Pant C, Anderson MP, Donskey CJ, Sferra TJ. Clostridium difficile infection in the hospitalized pediatric population: increasing trend in disease incidence. Pediatr Infect Dis J 2013; 32: 11381140. https://doi.org/10.1097/INF.0b013e3182948518

3. Lessa FC, Mu Y, Bamberg WM, et al. Burden of Clostridium difficile infection in the United States. $N$ Engl J Med 2015; 372: 825-834. https://doi. org/10.1056/NEJMoa1408913

4. Karaaslan A, Soysal A, Yakut N, et al. Hospital acquired Clostridium difficile infection in pediatric wards: a retrospective case-control study. Springerplus 2016; 5: 1329. https://doi.org/10.1186/ s40064-016-3013-8

5. Dubberke ER, Olsen MA. Burden of Clostridium difficile on the healthcare system. Clin Infect Dis 2012; 55(Suppl 2): S88-S92. https://doi.org/10.1093/ $\mathrm{cid} / \operatorname{cis} 335$

6. Tabak YP, Zilberberg MD, Johannes RS, Sun X, McDonald LC. Attributable burden of hospital-onset Clostridium difficile infection: a propensity scores matching study. Infect Control Hosp Epidemiol 2013; 34: 588-596. https://doi.org/10.1086/670621 
7. Mehrotra P, Jang J, Gidengil C, Sandora TJ. Attributable cost of Clostridium difficile infection in pediatric patients. Infect Control Hosp Epidemiol 2017; 38: 1472-1477. https://doi.org/10.1017/ ice. 2017.240

8. McDonald LC, Gerding DN, Johnson S, et al. Clinical Practice Guidelines for Clostridium difficile infection in adults and children: 2017 update by the Infectious Diseases Society of America (IDSA) and Society for Healthcare Epidemiology of America (SHEA). Clin Infect Dis 2018; 66: e1-e48. https://doi. org/10.1093/cid/cix1085

9. Jangi S, Lamont JT. Asymptomatic colonization by Clostridium difficile in infants; implications for disease in later life. J Pediatr Gastroenterol Nutr 2010; 51: 2-7. https://doi.org/10.1097/ MPG.0b013e3181d29767

10. Enoch DA, Butler MJ, Pai S, Aliyu SH, Karas JA. Clostridium difficile in children: colonisation and disease. J Infect 2011; 63: 105-113. https://doi. org/10.1016/j.jinf.2011.05.016

11. Lees EA, Miyajima F, Pirmohamed M, Carrol ED. The role of Clostridium difficile in the paediatric and neonatal gut- a narrative review. Eur J Clin Microbiol Infect Dis 2016; 35: 1047-1057. https://doi. org/10.1007/s10096-016-2639-3

12. Jangi S, Lamont JT. Asymptomatic colonization by Clostridium difficile in infants implications for disease in later life. J Pediatr Gastroenterol Nutr 2020; 51: 2-7. https://doi.org/10.1097/ MPG.0b013e3181d29767
13. Kukla M, Adrych K, Dobrowolska A, Mach T, Reguła J, Rydzewska G. Guidelines for Clostridium difficile infection in adults. Prz Gastroenterol 2020; 15: 1-21. https://doi.org/10.5114/pg.2020.93629

14. Kim J, Shaklee JF, Smathers S, et al. Risk factors and outcomes associated with severe Clostridium difficile infection in children. Pediatr Infect Dis J 2012; 31: 134-138. https://doi.org/10.1097/ INF.0b013e3182352e2c

15. Nicholson MR, Thomsen IP, Slaughter JC, Creech $\mathrm{CB}$, Edwards KM. Novel risk factors for recurrent Clostridium difficile infection in children. J Pediatr Gastroenterol Nutr 2015; 60: 18-22. https://doi. org/10.1097/MPG.0000000000000553

16. Khalil A, Hendaus MA, Elmagboul E, Mohamed A, Deshmukh A, Elmasoudi A. Incidence of Clostridium difficile infection and associated risk factors among hospitalized children in Qatar. Ther Clin Risk Manag 2019; 15: 1343-1350. https://doi. org/10.2147/TCRM.S229540

17. Nylund CM, Goudie A, Garza JM, Fairbrother G, Cohen MB. Clostridium difficile infection in hospitalized children in the United States. Arch Pediatr Adolesc Med 2011; 165: 451-457. https://doi. org/10.1001/archpediatrics.2010.282

18. Tai E, Richardson LC, Townsend J, Howard E, Mcdonald LC. Clostridium difficile infection among children with cancer. Pediatr Infect Dis J 2011; 30: 610612. https://doi.org/10.1097/INF.0b013e31820970d1 